\title{
Biomineralization of barnacle base plate in association with adhesive cement protein
}

\author{
SUNYOUNG HUR ${ }^{1,2}$, SOUHIR BOUJDAY ${ }^{1}$ AND ALI \\ MISEREZ $^{2}$ \\ ${ }^{1}$ Sorbonne Universite \\ ${ }^{2}$ Nanyang Technological University \\ Presenting Author: sunyoung.hur@sorbonne-universite.fr
}

The adhesive layer underneath the acorn barnacle Megabalanus rosa (Mr) is an attracting bioinspired system of both biomineralization and biological adhesive due to its functional roles at the interface adjacent to both the barnacle's own calcareous base plate and the external substrate which it adheres to. Growth of the barnacle includes expanding its base plate and periphery shell by molting and calcification, and at the same time secreting the adhesive proteins ${ }^{1}$. Nano- and microcrystalline $\mathrm{CaCO}_{3}$ of the base plate ${ }^{2}$, as well as the $3 \mathrm{D}$ structure of the adhesive protein "Mr20" ${ }^{3}$ have been studied so far, but detailed mechanisms of their interplay occurring at the submerged interface is yet unclear. Our work aims to understand how Mr20 may regulate calcium carbonate crystallization of the base plate, by studying: (1) protein-mediated crystal growth kinetics and morphology; and (2) the restructuring of the flexible Mr20 in the presence of mineral ions and during mineralization. The results demonstrate a mutual influence between the protein and mineral growth, which support a recent molecular dynamic modelling of their interplay ${ }^{4}$. This research brings insights into understanding the multifunctional roles of barnacle's adhesive protein underneath the base plate, and provides biomimetic lessons for the development of new bioinspired adhesives.

References:

[1] Barnacle shells: composition, structure and growth, Bourget, E. (1987), Barnacle biology 5, 267-285.

[2] Shell Structure and Growth in the Base Plate of the Barnacle Amphibalanus Amphitrite, Bradley T. De Gregorio et al. (2015), ACS Biomater. Sci. Eng. 1, 1085-1095.

[3] Three-dimensional structure of Megabalanus rosa Cement Protein 20 revealed by multi-dimensional NMR and molecular dynamics simulations, Mohanram et al. (2019), Phil. Trans. R. Soc. B 374: 20190198.

[4] Disorder-order Interplay of a Barnacle Cement Protein Triggered by Interactions with Calcium and Carbonate Ions: A Molecular Dynamics Study, Kumar et al. (2020), Chem. Mater. $32,8845-8859$.

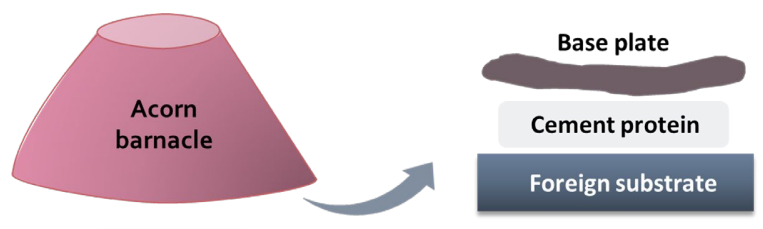

